\title{
Phase-space window and degrees of freedom of optical systems with multiple apertures
}

\author{
Haldun M. Ozaktas ${ }^{1}$ and Figen S. Oktem ${ }^{2, *}$ \\ ${ }^{1}$ Department of Electrical Engineering, Bilkent University, Bilkent, Ankara TR-06800, Turkey \\ ${ }^{2}$ Department of Electrical and Computer Engineering, University of Illinois at Urbana-Champaign, \\ Urbana, Illinois 61801, USA \\ ${ }^{*}$ Corresponding author: oktem1@illinois.edu
}

Received October 26, 2012; revised February 21, 2013; accepted February 22, 2013; posted February 22, 2013 (Doc. ID 178682); published March 21, 2013

\begin{abstract}
We show how to explicitly determine the space-frequency window (phase-space window) for optical systems consisting of an arbitrary sequence of lenses and apertures separated by arbitrary lengths of free space. If the space-frequency support of a signal lies completely within this window, the signal passes without information loss. When it does not, the parts that lie within the window pass and the parts that lie outside of the window are blocked, a result that is valid to a good degree of approximation for many systems of practical interest. Also, the maximum number of degrees of freedom that can pass through the system is given by the area of its spacefrequency window. These intuitive results provide insight and guidance into the behavior and design of systems involving multiple apertures and can help minimize information loss. (c) 2013 Optical Society of America
\end{abstract} OCIS codes: $\quad 070.0070,050.5082,070.2575,070.2590,080.2730,110.1220$.

\section{INTRODUCTION}

We propose a simple method to answer the following questions about apertured optical systems, which here refers to systems consisting of an arbitrary sequence of thin lenses and apertures separated by sections of free space: (a) Given the space-frequency support of an input signal and the parameters of an apertured optical system, will there be any information loss upon passage through the system? (b) Which set of signals can pass through a given apertured system without any information loss? In other words, what is the largest space-frequency support that can pass through the system without any information loss? (c) What is the maximum number of spatial degrees of freedom that can be supported by a given apertured system? The space-frequency support (phase-space support) of a set of signals may be defined as the region in the space-frequency plane (phase space) in which a large percentage of the total energy is confined $[1,2]$. The number of degrees of freedom is given by the area of the space-frequency support. We also define the spacefrequency window (phase-space window) of a system as the largest space-frequency support that can pass through the system without any information loss. Here we develop a simple method to find the space-frequency window of a given system in terms of its parameters. Once the spacefrequency window of the system is determined, it specifies the set of all signals that can pass through the system without information loss: the optical system preserves the information content of signals whose space-frequency supports lie inside the system window. All we need to do is to compare the spacefrequency support of the input signal with the space-frequency window of the system. If the signal support lies completely inside the system window, the signal will pass through the system without any information loss. Otherwise, information loss will occur.
The number of degrees of freedom of the set of signals that can pass through a system can be determined from the area of the space-frequency window of the system. Although the space-frequency window may in general have different shapes $[1,2]$, it is often assumed to be of rectangular shape with the spatial extent determined by a spatial aperture in the object or image plane, and the frequency extent determined by an aperture in a Fourier plane. We consider one-dimensional signals and systems for simplicity. If these apertures are of length $\Delta x$ and $\Delta \sigma_{x}$ respectively, then the number of degrees of freedom that can be supported by the system is given by $\Delta x \Delta \sigma_{x}$. More generally, for space-frequency windows of different (nonrectangular) shapes, the number of degrees of freedom is given by the area of the space-frequency window.

Physical systems that carry or process signals always limit their spatial extents and bandwidths to certain finite values. A physical system cannot allow the existence of frequencies outside a certain band because there is always some limit to the resolution that can be supported. Likewise, since all physical events of interest have a beginning and an end, or since all physical systems have a finite extent, the temporal duration or spatial extent of the signals will also be finite. For example, in an optical system the sizes of the lenses will limit both the spatial extent of the images that can be dealt with and their spatial bandwidths. More generally, we may say that they will limit the signal to a certain region in the space-frequency plane. We refer to this region as the space-frequency window of the system. It is these physical limitations that determine the space-frequency support of the signals and thus their degrees of freedom. Just as these may be undesirable physical limitations that limit the performance of the system, they may also be deliberate limitations with the purpose of limiting the set of signals we are dealing with. When a signal previously represented by a system with larger space-frequency window 
is input into a system with smaller space-frequency window, information loss takes place.

The conventional space-bandwidth product has been of fundamental importance because of its interpretation as the number of degrees of freedom [1-17]. In most works, the space-bandwidth product, as its name implies, is the product of a spatial extent and a spatial-frequency extent. This implies the assumption of a rectangular space-frequency region. However, the set of input signals may not exhibit a rectangular space-frequency support, and even if they do, this support will not remain rectangular as it propagates through the system [18-20]. Likewise, the space-frequency windows of multicomponent optical systems, as we will see in this paper, do not in general exhibit rectangular shapes. This possibility and some of its implications were discussed in [2]. In this paper we make concrete the hypothetical concept of a nonrectangular space-frequency window, and show how it can be actually computed for a broad class of optical systems. To prevent possible confusion, we emphasize that we are dealing with systems with sequentially cascaded apertures, and not systems with multiple parallel apertures.

Lastly, we note that the phase-space window has been referred to by different names, such as the space-bandwidth product of the system (in short SWY) $[2,21,22]$, the system transmission range [21], and the Wigner or space-bandwidth chart of the system [21,22]. Also, the concept of degrees of freedom can be related to other concepts such as Shannon number and information capacity of an optical system [16], geometrical etendue [23], dimensionality, and so on.

We first review quadratic-phase systems and then discuss how to find the phase-space window of a system. We then treat the cases of lossless and lossy transfer separately, and finally conclude with a discussion of applications. This work is based on [20].

\section{QUADRATIC-PHASE SYSTEMS}

Optical systems involving thin lenses, sections of free space in the Fresnel approximation, sections of quadratic graded-index media, and arbitrary combinations of any number of these are referred to as (lossless) first-order optical systems or quadratic-phase systems ( $A B C D$ systems). Mathematically, such systems can be modeled as linear canonical transforms (LCTs), which are a three-parameter family of linear integral transforms [24-29]. The output $f_{\mathrm{T}}(x)$ of a quadratic-phase system is related to its input $f(x)$ through $[\underline{1}, \underline{30}, \underline{31}]$

$$
\begin{aligned}
f_{\mathbf{T}}(x) & \equiv\left(\mathcal{C}_{\mathbf{T}} f\right)(x) \equiv \int_{-\infty}^{\infty} C_{\mathbf{T}}\left(x, x^{\prime}\right) f\left(x^{\prime}\right) \mathrm{d} x^{\prime}, \\
C_{\mathbf{T}}\left(x, x^{\prime}\right) & \equiv \sqrt{\frac{1}{B}} e^{-i \pi / 4} e^{i \pi\left(\frac{D}{B} x^{2}-2 \frac{1}{B} x x^{\prime}+\frac{A}{B} x^{\prime 2}\right)}
\end{aligned}
$$

for $B \neq 0$, where $\mathcal{C}_{\mathbf{T}}$ is the unitary LCT operator with system parameter matrix $\mathbf{T}=[A B ; C D]$ and $A D-B C=1$. In the trivial case $B=0$, the output is related to the input as $f_{\mathrm{T}}(x) \equiv \sqrt{D} \exp \left(i \pi C D x^{2}\right) f(D x)$. Sometimes the three real parameters $\alpha=D / B, \beta=1 / B$, and $\gamma=A / B$ are used instead of the unit-determinant matrix $\mathbf{T}$, whose elements are $A, B, C$, and $D$. One of the four matrix parameters is redundant because of the unit-determinant condition. The transformation matrix $\mathbf{T}$ is useful in the analysis of optical systems because if several systems are cascaded, the overall system matrix can be found by multiplying the corresponding matrices. Fourier and fractional Fourier transforms (FRTs) (propagation through quadratic graded-index media), coordinate scaling (imaging), chirp multiplication (passage through a thin lens), and chirp convolution (Fresnel propagation in free space) are some of the special cases of quadratic-phase systems.

The $a$ th-order FRT [1] of a function $f(x)$, denoted by $f_{a}(x)$, is commonly defined as

$$
\begin{aligned}
f_{a}(x) & \equiv\left(\mathcal{F}^{a} f\right)(x) \equiv \int_{-\infty}^{\infty} K_{a}\left(x, x^{\prime}\right) f\left(x^{\prime}\right) \mathrm{d} x^{\prime}, \\
K_{a}\left(x, x^{\prime}\right) & \equiv \frac{A_{\phi}}{s} e^{i \pi\left(\frac{\cot \phi}{s^{2}} x^{2}-2 \frac{\csc \phi}{s^{2}} x x^{\prime}+\frac{\cot \phi}{s^{2}} x^{\prime 2}\right)}, \\
A_{\phi} & =\sqrt{1-i \cot \phi}, \quad \phi=a \pi / 2
\end{aligned}
$$

when $a \neq 2 k, \quad K_{a}\left(x, x^{\prime}\right)=\delta\left(x-x^{\prime}\right) \quad$ when $a=4 k$, and $K_{a}\left(x, x^{\prime}\right)=\delta\left(x+x^{\prime}\right)$ when $a=4 k \pm 2$, where $k$ is an integer. The FRT operator $\mathcal{F}^{a}$ is additive in index, $\mathcal{F}^{a_{2}} \mathcal{F}^{a_{1}}=\mathcal{F}^{a_{2}+a_{1}}$, and reduces to the Fourier transform (FT) and identity operators for $a=1$ and $a=0$, respectively. The FRT is a special case of the LCT with parameter matrix

$$
\mathbf{F}^{a}=\left[\begin{array}{cc}
\cos (a \pi / 2) & s^{2} \sin (a \pi / 2) \\
-\frac{\sin (a \pi / 2)}{s^{2}} & \cos (a \pi / 2)
\end{array}\right]
$$

differing only by an inconsequential factor: $\mathcal{C}_{\mathbf{F}^{a}} f(x)=$ $e^{-i a \pi / 4} \mathcal{F}^{a} f(x)[\underline{1}, 31]$. Here $s$ is an arbitrary scale parameter with dimensions of length. The scale parameter serves to convert the dimensional terms inside the integral to dimensionless form. The FRT definition above reduces to the pure mathematical FRT definition with dimensionless arguments if we define the dimensionless variables $u=x / s$ and $u^{\prime}=x^{\prime} / s$, or simply if we set $s=1$ in our measurement unit (meters, etc.). The choice $s=1$ unit makes the expressions simpler, but we feel that this merely hides the essential distinction between dimensional and dimensionless variables and would actually be a disservice to the reader.

An arbitrary quadratic-phase system can be decomposed into a (dimensional) FRT followed by scaling, followed by chirp multiplication $[\underline{1}, \underline{32}, \underline{33}]$ :

$$
\mathbf{T}=\left[\begin{array}{ll}
A & B \\
C & D
\end{array}\right]=\left[\begin{array}{cc}
1 & 0 \\
-\frac{q}{s^{2}} & 1
\end{array}\right]\left[\begin{array}{cc}
M & 0 \\
0 & \frac{1}{M}
\end{array}\right]\left[\begin{array}{cc}
\cos \phi & s^{2} \sin \phi \\
-\frac{\sin \phi}{s^{2}} & \cos \phi
\end{array}\right]
$$

The three matrices correspond to the transformation matrices of chirp multiplication with parameter $q$ [multiplication by $\exp \left(-i \pi\left(q / s^{2}\right) x^{2}\right)$ ], coordinate scaling with factor $M>0$ [mapping of $f(x)$ into $\sqrt{1 / M} f(x / M)]$, and ath-order FRT with $\phi=$ $a \pi / 2$ [transformation of $f(x)$ into $f_{a}(x)$ ], respectively. The decomposition can be written more explicitly in terms of the LCT- and FRT-domain representations of the signal as

$$
f_{\mathbf{T}}(x)=\exp \left(-i \pi \frac{q}{s^{2}} x^{2}\right) \sqrt{\frac{1}{M}} f_{a}\left(\frac{x}{M}\right)
$$

This decomposition is a special case of the Iwasawa decomposition [34-36]. (For a discussion of the implications of this decomposition on the propagation of light through first-order optical systems, see [19,37]. For a discussion of the implications for sampling optical fields, see [38,39].) By appropriately 
choosing the three parameters $a, M$, and $q$, the above equality can be satisfied for any $\mathbf{T}=[A B ; C D]$ matrix. Solving for $a, M$, and $q$ in Eq. (4), we obtain the decomposition parameters in terms of the matrix entries $A, B, C$, and $D$ :

$$
\begin{gathered}
a=\left\{\begin{array}{ll}
\frac{2}{\pi} \arctan \left(\frac{1}{s^{2}} \frac{B}{A}\right), & \text { if } A \geq 0 \\
\frac{2}{\pi} \arctan \left(\frac{1}{s^{2}} \frac{B}{A}\right)+2, & \text { if } A<0
\end{array},\right. \\
M=\sqrt{A^{2}+\left(B / s^{2}\right)^{2},} \\
q=\left\{\begin{array}{ll}
-s^{2} \frac{C}{A}-\frac{1}{s^{2}} \frac{B / A}{A^{2}+\left(B / s^{2}\right)^{2}}, & \text { if } A \neq 0 \\
-s^{2} \frac{D}{B}, & \text { if } A=0
\end{array} .\right.
\end{gathered}
$$

The range of the arctangent lies in $(-\pi / 2, \pi / 2]$.

The Wigner distribution (WD) $W_{f}\left(x, \sigma_{x}\right)$ of a signal $f(x)$ is a space-frequency (phase-space) distribution that gives the distribution of signal energy over space and frequency, and is defined as $[\underline{1, \underline{40}-\underline{42}]}$

$$
W_{f}\left(x, \sigma_{x}\right)=\int_{-\infty}^{\infty} f\left(x+x^{\prime} / 2\right) f^{*}\left(x-x^{\prime} / 2\right) e^{-i 2 \pi \sigma_{x} x^{\prime}} \mathrm{d} x^{\prime} .
$$

We refer to the space-frequency region for which the WD is considered nonnegligible as the space-frequency support of the signal, with the area of this region giving the number of degrees of freedom [1]. All quadratic-phase systems result in an area-preserving geometric transformation in the $x-\sigma_{x}$ plane. Explicitly, the WD of $f_{\mathrm{T}}(x)$ can be related to the WD of $f(x)$ by a linear distortion [1]:

$$
W_{f_{\mathbf{T}}}\left(x, \sigma_{x}\right)=W_{f}\left(D x-B \sigma_{x},-C x+A \sigma_{x}\right) .
$$

The Jacobian of this coordinate transformation is equal to the determinant of the matrix $\mathbf{T}$, which is unity. Therefore, this coordinate transformation will geometrically distort the support region of the WD, but the support area will remain unchanged.

\section{PHASE-SPACE WINDOW OF OPTICAL SYSTEMS}

We now describe how to find the phase-space window (spacefrequency window) of an apertured optical system. Such systems consist of arbitrary concatenations of apertures with quadratic-phase systems, which in turn consist of an arbitrary number of lenses, sections of free space, and quadratic graded-index media. Also note that a lens with a finite aperture can be viewed as an ideal lens followed by a finite aperture. Although beyond the scope of this paper, our results can be extended to more general systems involving occlusions [43], prisms and gratings [22], and bends and shifts of the optical axis.

The input and output planes are defined along the optical axis $z$ at $z=0$ and $z=d$, where $d$ is the length of the system. If the apertures did not exist, the amplitude distribution at any plane perpendicular to the optical axis could be expressed as an LCT of the input. Hence each $z$ plane corresponds to an LCT domain. Let $L$ denote the total number of apertures in the system. $z_{j}$ and $\Delta_{j}$ will denote the location and extent of the $j$ th aperture in the system, $j=1,2, \ldots, L$. The matrix $\mathbf{T}_{j}$ will be used to denote the parameter matrix of the system from the input to the position of the $j$ th aperture-that is, the system lying between 0 and $z_{j}$ excluding the apertures. The matrix $\mathbf{T}_{j}$ can be readily calculated using the matrices for lenses, sections of free space, quadratic graded-index media, and the concatenation property [1]. The matrix elements of $\mathbf{T}_{j}$ will be denoted by $A_{j}, B_{j}, \bar{C}_{j}$, and $D_{j}$. The associated Iwasawa decomposition parameters will be denoted by $a_{j}, M_{j}$, and $q_{j}$, which can be computed from $A_{j}$, $B_{j}, C_{j}$, and $D_{j}$ by using formulas ( $\left.\underline{6}\right)-(\underline{8})$. The FRT order in the Iwasawa decomposition begins from 0 at the input of the system, and then monotonically increases as a function of distance [1,19].

For lossless transfer through the system, the extent of the signal just before each aperture must lie inside the aperture. For simplicity we assume that both the aperture and the signal extents are centered around the origin. Then, the following must be satisfied for $j=1,2, \ldots, L$ :

$$
\Delta x_{\mathbf{T}_{j}} \leq \Delta_{j},
$$

where $\Delta x_{\mathbf{T}_{j}}$ denotes the extent of the signal in the $x_{\mathbf{T}_{j}}$ domain, which corresponds to the LCT domain at the $z=z_{j}$ plane, where the $j$ th aperture is situated.

It is well known that the $a$ th-order FRT domain is an oblique axis in the space-frequency plane $[\underline{1,44}]$. It has recently been shown that LCT domains are equivalent to scaled FRT domains and thus to scaled oblique axes in the space-frequency plane [19]. The equivalence between LCT and FRT domains is based on expressing the LCT as a chirp-multiplied and scaled FRT, as given in Eq. (5). Multiplication is not considered to be an operation that changes the domain of a signal, and scaling of the axis is a relatively trivial modification of a domain. Therefore, the only part of the LCT operation that genuinely corresponds to a domain change is the FRT in it, and the linear canonical transformed signal essentially lives in a scaled fractional Fourier domain. With this equivalence, each finite interval in an LCT domain will correspond to a scaled interval in the equivalent FRT domain. To see this explicitly, we again refer to Eq. (5), which implies that if the linear canonical transformed signal $f_{\mathrm{T}}(x)$ is confined to an interval of length $\Delta x_{\mathrm{T}}$, so is $f_{a}(x / M)$. Therefore, the extent of the fractional Fourier transformed signal $f_{a}(x)$ in the equivalent FRT domain is $\Delta x_{\mathbf{T}} / M$. Thus, the condition in Eq. (11) can be reexpressed as

$$
\Delta x_{a_{j}} \leq \Delta_{j} / M_{j},
$$

where $\Delta x_{a_{j}}$ denotes the extent of the signal in the $a_{j}$ th-order (dimensional) FRT domain.

FRT domains are often visualized in the dimensionless space-frequency plane where the coordinates are scaled such that the space and frequency axes are dimensionless. This is achieved by introducing the scaling parameter $s$ and the dimensionless scaled coordinates $u=x / s$ and $\mu=s \sigma_{x}$. The condition for lossless information transfer then becomes

$$
\Delta x_{a_{j}} / s \leq \Delta_{j} / M_{j} s,
$$

where $\Delta x_{a_{j}} / s$ denotes the extent of the signal along the oblique axis, making angle $a_{j} \pi / 2$ with the $u=x / s$ axis. 
In other words, for every $j=1,2, \ldots, L$, the signal must be confined to the interval of length $\Delta_{j} / M_{j} s$ in the $a_{j}$ th-order FRT domain along the oblique axis with angle $a_{j} \pi / 2$.

It is well known that if the space-, frequency-, or FRTdomain representation of a signal is identically zero (or negligible) outside a certain interval, so is its WD [1,ㅎ5]. As a direct consequence of this fact, the condition in Eq. (13) defines a corridor of width $\Delta_{j} / M_{j} s$ in the direction orthogonal to the $a_{j}$ th-order FRT domain $u_{a_{j}}$. With the term "corridor" we are referring to an infinite strip in the space-frequency plane perpendicular to the oblique $u_{a_{j}}$ axis. The corridor makes an angle $\left(a_{j}+1\right) \pi / 2$ with the $u=x / s$ axis in the dimensionless space-frequency plane (see Fig. 1). Now, if we intersect the corridors defined by each aperture, we obtain a bounded region in the space-frequency plane, which has the form of a centrally symmetrical convex polygon (see Fig. 2 for $L=2$ and Fig. 3 for $L=4$ ). We will refer to this convex polygon defined by the normalized aperture extents as the spacefrequency window of the system, in dimensionless space.

The space-frequency window specifies the set of all signals that can pass through the system without any loss: the optical system preserves the information content of all signals whose supports lie inside the space-frequency window. The area of the space-frequency window gives the number of degrees of freedom that can pass through the system. This is also the minimum number of samples required to faithfully represent an arbitrary signal at the output of the system.

We can summarize the steps for finding the phase-space window (space-frequency window) as follows:

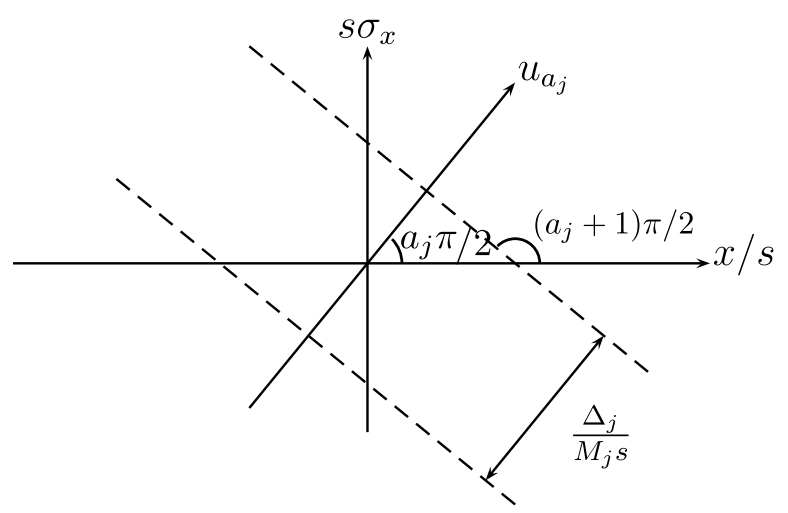

Fig. 1. Illustration of a space-frequency corridor.

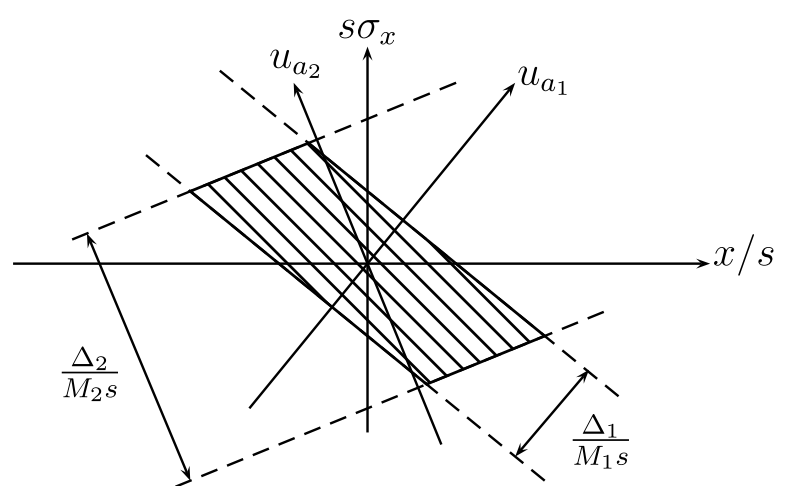

Fig. 2. Space-frequency window of a system with two apertures.

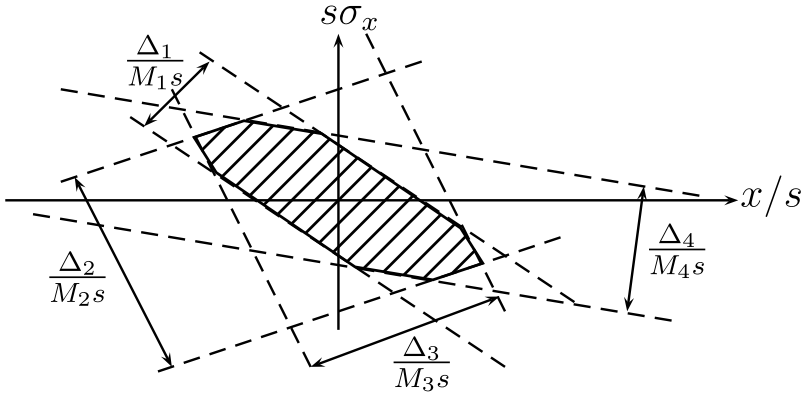

Fig. 3. Space-frequency window of a system with four apertures.

1. Compute the parameter matrix $\mathbf{T}_{j}$ for each aperture $j=1,2, \ldots, L$ using the matrices for lenses, sections of free space and quadratic graded-index media, and the concatenation property. Recall that $\mathbf{T}_{j}$ was defined as the parameter matrix of the system lying between the input plane and the location of the $j$ th aperture.

2. Compute the corresponding Iwasawa decomposition parameters $a_{j}$ and $M_{j}$ (the fractional order and the magnification) by inserting the matrix entries $A_{j}, B_{j}, C_{j}$, and $D_{j}$ into formulas (6) and (7).

3. In the dimensionless space-frequency plane, draw a corridor of width $\Delta_{j} / M_{j} s$ making angle $\left(a_{j}+1\right) \pi / 2$ with the $x / s$ axis, for each $j$. The corridor is explicitly defined by the following two lines: $y=-\cot \left(a_{j} \pi / 2\right) x \pm \frac{\Delta_{j}}{2 M_{j} s} \csc \left(a_{j} \pi / 2\right)$.

4. Intersect the corridors from all apertures to determine the region lying inside all the corridors. This is the phasespace window at the input plane $z=0$.

5. Scale the horizontal and vertical coordinates by $s$ and $1 / s$, respectively, to obtain the phase-space window in the dimensional space-frequency plane $x-\sigma_{x}$.

A few remarks are in order at this point. First, the area of the window and hence the number of degrees of freedom of the system remains the same whether it is computed in dimensional or dimensionless space. Second, choice of the scale parameter $s$ is arbitrary and the system window in the dimensional space-frequency plane is independent of the choice of $s$. However, choice of $s$ does affect the value of $a$ as a function of $z$. Some choices better utilize the range of $a$ (as in Fig. 4), whereas poor choices lead to $a$ changing too quickly over a short range of $z$ and then saturating [1, pp. 320-321 and 377-378]. One approach is to choose $s$ such that the space and frequency extents in the dimensionless space-frequency plane are comparable to each other. Third, the system window is computed with respect to a chosen reference plane. Above, we compute it with respect to the input plane, so that we can compare the input signal support with the system window. If one desires to visualize the system window with respect to a different reference plane, it can be transformed to the new plane using the LCT transformation from the input plane to the new reference plane.

We now illustrate the method on a sample system. Figure 4(a) shows a system consisting of several apertures and lenses, whose aperture sizes and focal lengths are given right above them. The fractional transform order $a$ and the scale parameter $M$ of the system are plotted in Fig. $\underline{4}$ as functions of distance $z$. The emphasis in this paper is on computing $a_{j}$ and $M_{j}$ at the aperture locations, since these allow us to determine whether there is any information loss. However, 


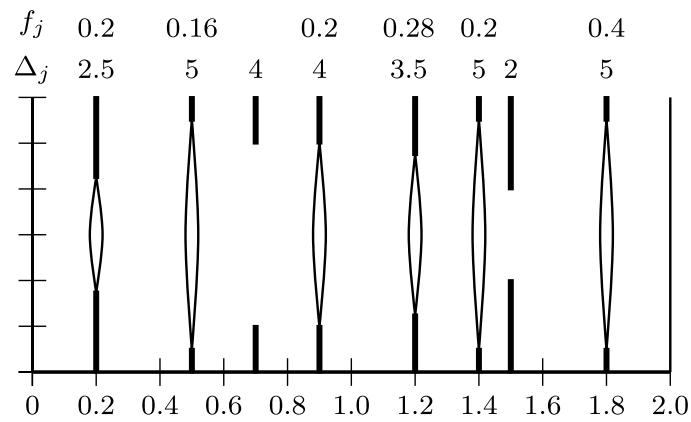

(a)

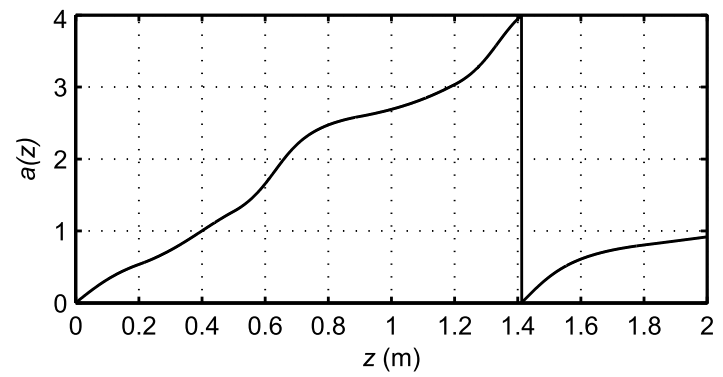

(b)

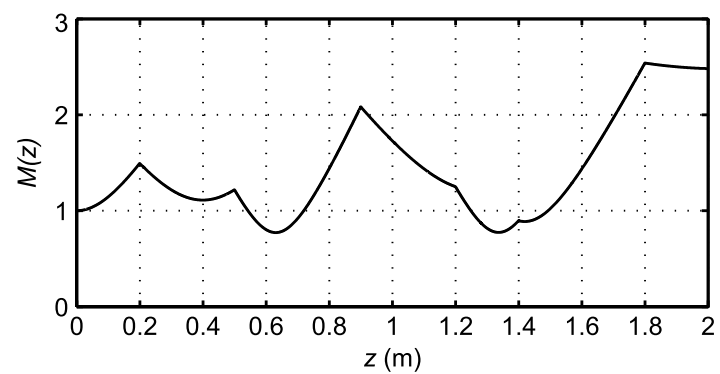

(c)

Fig. 4. (a) Apertured optical system with input plane at $z=0$ and output plane at $z=2 \mathrm{~m}$. The horizontal axis is in meters. The lens focal lengths $f_{j}$ in meters and the aperture sizes $\Delta_{j}$ in centimeters are given right above them. (b) and (c) Evolution of $a(z)$ and $M(z)$ as functions of $z \cdot \lambda=0.5 \mu \mathrm{m}$ and $s=0.3 \mathrm{~mm}[1,37]$.

these quantities can also be computed for all values of $z$ in the system, revealing their continual evolution as we move along the optical axis, as illustrated in Figs. $\underline{4(\mathrm{~b})}$ and $4(\mathrm{c})$. We can

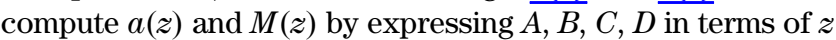
and using them in Eqs. (6)-(8) [1,37].

Figure 5 shows the system window at the input plane $z=0$. This region defines the set of all input signals that can pass through the system without any information loss. Input signals whose space-frequency support lies wholly inside this region will not experience any loss. Similarly, Fig. 6 shows the system window at the output plane $z=d$. This region defines the set of all signals that can be observed at the output of the system. The region in Fig. $\underline{6}$ is just a propagated version of the region in Fig. $\underline{5}$ through the whole optical system. This can be obtained by applying the concatenated LCT matrix $\mathbf{T}_{L}, \ldots, \mathbf{T}_{2} \mathbf{T}_{1}$ to the space-frequency window at the input plane, to take into account the linear distortion due to the whole optical system.

Just as the concatenation property of transformation matrices allows us to represent the cumulative action of all optical elements present with a single entity, the system window is

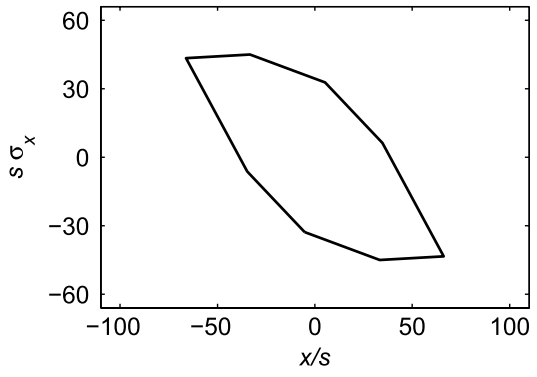

(a)

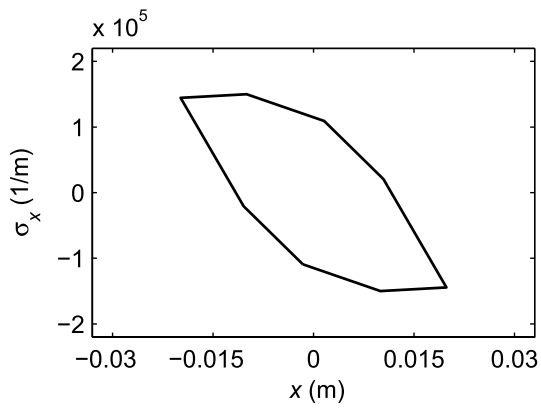

(b)

Fig. 5. Space-frequency window of the system at the input plane in the dimensionless (a) and dimensional (b) spaces.

an equivalent aperture that appropriately transforms and combines the effects of all individual apertures in different domains into a single space-frequency aperture.

The space-frequency (phase-space) window of the system in Fig. 4(a) is determined only by the first, fifth, seventh, and eighth apertures. The other apertures do not affect or limit the spacefrequency window of the system and therefore can be considered as redundant from the system's viewpoint. Removing the redundant apertures from the system or replacing them with

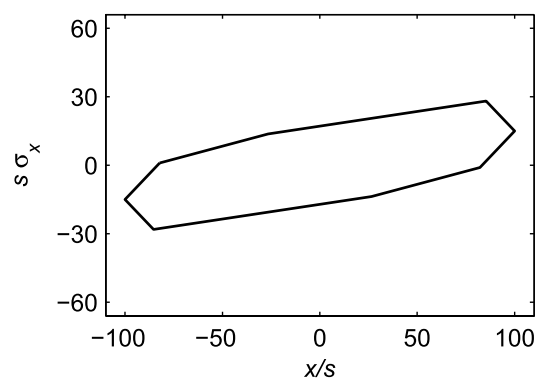

(a)

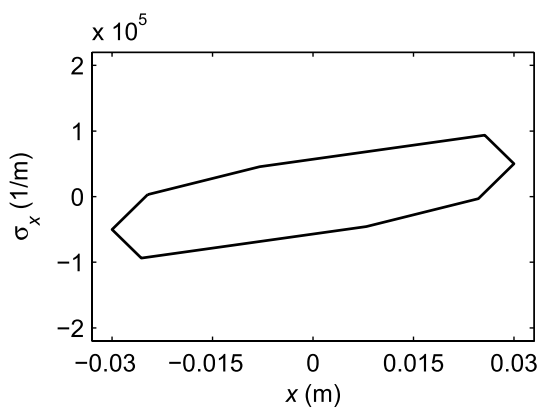

(b)

Fig. 6. Space-frequency window of the system at the output plane in the dimensionless (a) and dimensional (b) spaces. 
apertures of greater size will have negligible effect on the behavior of the system, for any given input signal.

It is also worth noting that the information loss caused by an aperture will depend not only on the actual physical size of the aperture, but also on the magnification of the signal at that location. If the magnification at the aperture location is small, there will be less or no information loss. For example, although the aperture sizes are the same for the second, sixth, and eighth apertures, only the eighth aperture limits the system window $[M(1.8) \approx 2.5$, whereas $M(0.5) \approx 1.2$ and $M(1.4) \approx 1]$. This illustrates that the magnification in the plane of the aperture is as important as the size of the aperture in limiting the system window. If we have some flexibility during the design of the optical system, careful choice of lens and aperture locations can help information losses to be minimized, a process that will be aided by the space-frequency approach and the graphs for $M(z)$ we have discussed.

\section{NECESSARY AND SUFFICIENT CONDITION FOR LOSSLESS TRANSFER}

An input signal will pass through the system without any information loss if and only if its space-frequency support is fully contained in the space-frequency (phase-space) window of the system. That is, if the signal support does not lie completely inside the system window, information loss will occur.

Proof. LCT domains correspond to oblique axes in the space-frequency plane. Consider corridors of varying width, orthogonal to such an oblique axis. The extent of the signal in a given LCT domain can be determined from the spacefrequency support of the signal, by finding the width of the narrowest orthogonal corridor enclosing the space-frequency support. First, let us consider an input signal whose spacefrequency support lies completely inside the space-frequency window of the system. This guarantees that along any oblique axis in the space-frequency plane corresponding to some LCT domain in which an aperture resides, the extent of the signal will be smaller than the width of the aperture at that LCT domain, and hence the signal will pass through that aperture unhindered. Recall that the space-frequency window is defined by the intersection of the corridors defined by the apertures. If the extent of the signal was larger than the width of the aperture at that LCT domain, the orthogonal corridor enclosing the space-frequency support would have been wider than the corridor defined by the aperture, so that the space-frequency support of the signal could not lie within the space-frequency window of the system.

Conversely, consider an input signal that passes through the system without any loss. This implies that the signal extent was smaller than the aperture width for each aperture, since otherwise irreversible information loss would occur. Recall that each aperture defines a corridor perpendicular to the LCT domain in which it resides. For any of these LCT domains, the space-frequency support of the signal must lie within this corridor, since if not, the extent of the signal in that domain would not lie within that aperture, leading to information loss and hence a contradiction. Since this argument must be true for all apertures, it follows that the signal spacefrequency support must lie inside the region defined by the intersection of the corridors, which is the space-frequency window of the system. This completes the proof.
A straightforward but lengthy way to determine whether information loss will take place would be to trace the space-frequency support of the signal as it passes through the whole system. When the signal arrives at the first aperture, there will have taken place a linear distortion on the initial space-frequency support of the signal. After this linear distortion, if the extent of the signal in that LCT domain is less than the aperture size, then the signal will pass through this aperture without any information loss. Then another linear distortion will take place as the signal travels to the next aperture. Again, we will determine whether there is any information loss by comparing the extent in this domain to the aperture size. Repeating this procedure throughout the system, we can determine whether the signal passes through the system losslessly. This lengthy way of determining whether there will be information loss is specific to a certain input signal and its support. On the other hand, our method is general in the sense that, once the space-frequency window of the system is determined, it specifies the set of all signals that can pass through the system without information loss. The optical system preserves the information content of all signals whose spacefrequency support lies inside the space-frequency window of the system.

\section{LOSSY TRANSFER}

If the space-frequency window does not enclose the spacefrequency support of the input signal completely, then we would intuitively expect the following: the information contained within the intersection of the space-frequency support of the signal and the space-frequency window of the system will be preserved, and the rest will be lost (Fig. 7). This indeed turns out to be approximately true in many cases. In other words, just as a spatial aperture passes certain parts of a signal and blocks the rest, the space-frequency window acts like

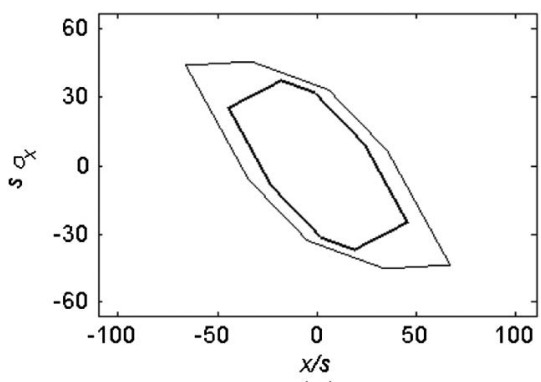

(a)

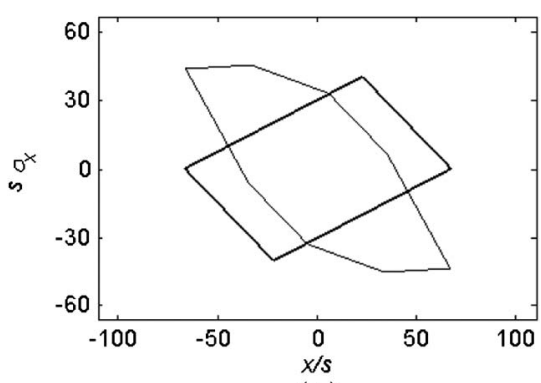

(b)

Fig. 7. (a) Signal support is wholly contained within the system window so there is no loss of information. (b) Part of the signal support lying within the system window will pass, and the parts lying outside will be blocked. 
an aperture in phase space, passing certain parts and blocking others. In particular, if the set of input signals has a greater number of degrees of freedom than the number of degrees of freedom the system can support, information loss will take place, since a region with larger area can never possibly lie completely within a region with smaller area.

Given an arbitrary space-frequency support at the input, one can obtain the space-frequency support at any position in the system by tracing the support throughout the system. Whenever an aperture narrower than the signal extent is encountered, the outlying parts of the signal will be truncated. The effect of this truncation on the space-frequency support of the signal will be to likewise truncate the regions of the support lying outside the corridor defined by the aperture. If this were the only effect of the aperture in the space-frequency plane, then the statements made above would be exact (rather than being approximate) and the space-frequency support observed at the output could simply be found as follows: (i) find the intersection of the input space-frequency support and the system space-frequency window and (ii) propagate this space-frequency region to the output plane. However, this simple and intuitive result is not exact because each aperture that actually cuts off the outlying parts of the signal will also cause a broadening of the support of the signal along the orthogonal domain, due to the Fourier uncertainty relation.

We now argue that the broadening effects are generally negligible for many real physical signals and systems, so that the simple and intuitive result above is often valid. The effect of an aperture corresponds to multiplication with a rectangle function. Let $\Delta_{j}$ denote the size of the aperture. First, if the signal extent before the aperture is already smaller than $\Delta_{j}$, then the windowing operation will affect neither the signal nor its space-frequency support. However, if the signal extent in that domain is larger than $\Delta_{j}$, then the signal will be truncated and the space-frequency support will also be affected. Because windowing involves multiplication with a rectangle function, it implies convolution of the WD of the signal with the WD of the rectangle function along the orthogonal direction $[40, \underline{45}]$. (An expression for the WD, $W_{\text {rect }}\left(x, \sigma_{x}\right)$ of the rectangle function $\operatorname{rect}\left(x / \Delta_{j}\right)$ is known $[\underline{1,46]}$, but its exact form is not necessary for our argument.) This operation will cause compaction of the WD of the signal to a corridor of width $\Delta_{j}$. Moreover, convolving the WD of the signal with that of the rectangle function along the orthogonal direction will result in broadening of the WD by an amount that is comparable with the extent of $W_{\text {rect }}$ in that direction. This extent is approximately $1 / \Delta_{j}$, and thus the spread in the orthogonal direction after windowing will be $\sim 1 / \Delta_{j}[\underline{46}]$.

For simplicity, consider a rectangular region in the spacefrequency plane, in which case the space-bandwidth product can be taken as a measure of the number of degrees of freedom. Let us denote the space-bandwidth product as $N=\Delta x \Delta \sigma_{x} \geq 1$, where $\Delta x$ and $\Delta \sigma_{x}$ denote the spatial and frequency extents. Noting that the apertures can be modeled as rectangle functions, the frequency extent associated with the rectangle function will approximately be the reciprocal of its spatial extent: $1 / \Delta_{j}$. Let us assume that the aperture extent is a fraction $\kappa$ of the signal extent; that is, $\Delta_{j}=\kappa \Delta x$, where $\kappa<1$. After the aperture, the new space-domain signal extent will be given by $\Delta x^{\prime}=\kappa \Delta x$. Moreover, since multiplication in the space-domain implies convolution in the frequency domain, the new extent in the frequency domain will be approximately the sum of the spectral extents of the signal and the aperture. The frequency extent of the signal is $\Delta \sigma_{x}=N / \Delta x$, and the frequency extent of the window is $\sim 1 / \Delta_{j}=1 / \kappa \Delta x=$ $\Delta \sigma_{x} / \kappa N$. Then, the new extent in the frequency domain will be $\Delta \sigma_{x}^{\prime} \approx \Delta \sigma_{x}+\Delta \sigma_{x} / \kappa N=\Delta \sigma_{x}(1+1 / \kappa N)$. Therefore, the space-bandwidth product of the signal after the aperture will be $\Delta x^{\prime} \Delta \sigma_{x}^{\prime} \approx \Delta x \Delta \sigma_{x}(\kappa+1 / N)$. Here, the first term corresponds to the reduced space-frequency support resulting from the truncation inflicted by the aperture, and the second term corresponds to the increase arising from the broadening in the orthogonal direction. However, if $\kappa \gg 1 / N$, or equivalently $N \gg 1 / \kappa$, then we can neglect the term $1 / N$ in comparison to $\kappa$. Thus, we can neglect the broadening effect if $N \gg 1 / \kappa$. This condition will hold for many real physical signals and systems. For a physical signal that contains any reasonable amount of information, such as an image, the number of degrees of freedom will be much larger than unity and also much larger than $1 / \kappa$, as long as $\kappa$ is not very close to 0 . The case where $\kappa$ is very close to 0 is not very likely either, since apertures with very small $\kappa$ truncate nearly all of the signal. For instance, consider a window that allows only 0.1 of the extent of the signal to pass. Even in this case, $N \gg 10$ will be sufficient and most information bearing signals will satisfy this condition easily. Therefore, the broadening effect will usually be negligible when we are dealing with images and other information bearing signals. This in turn means that it is fairly accurate to say that when the space-frequency support of such a signal does not wholly lie within the system window, the part that does lie within will pass, and the remaining parts will be lost. On the other hand, this simple result will not hold for some signals that do not exhibit too much spatial structure, such as a laser beam, and the broadening effect must be taken into account.

\section{CONCLUSION}

We have considered apertured optical systems, consisting of an arbitrary sequence of lenses and apertures separated by arbitrary lengths of free space (or quadratic graded-index media). We defined the space-frequency window (phasespace window) and showed how it can be explicitly determined for such a system. The area of the window gives the maximum number of degrees of freedom that can be supported by the system. More significantly, the window specifies which signals can pass through the system without information loss; we showed that the signal will pass losslessly if and only if the space-frequency support of the signal lies completely within this window. A precondition for lossless passage is of course that the area of the space-frequency support (and thus the number of degrees of freedom) of the set of input signals must be smaller than the area of the spacefrequency window (and thus the number of degrees of freedom the system can support). We further saw that when the space-frequency support does not lie completely within the space-frequency window, the parts that lie within the window pass and the parts that lie outside of the window are blocked. While the last result is not exact, we showed that it is valid to a good degree of approximation for many systems of practical interest.

Once established, these results are very intuitive and provide considerable insight and guidance into the behavior and 
design of systems involving multiple apertures. Our main contribution has been to show how the hypothetical concept of space-frequency windows can actually be calculated for a quite broad class of optical systems. Thus our results can help us design systems in a manner that minimizes information loss, for instance, by ensuring that the magnifications are as small as possible at aperture locations. An advantage of our approach is that it does not require assumptions regarding the signals during analysis or design, since the concept of a system window is signal-independent.

Being able to determine the space-frequency window as a function of the system parameters as we have shown, and the possibility of tailoring and optimizing it, has potential applications in areas including optical superresolution $[\underline{21,22}$, 47-51], holographic imaging [5, $\underline{43}, \underline{52}-55]$, optical design [56], optical encryption systems [57], analysis and design of recording devices $[\underline{16}, \underline{23}]$, and comparison between different implementations of a particular system [58], where apertured optical systems are involved.

In this paper we have mostly used the terms spacefrequency window or phase-space window to distinguish these entities living in the space-frequency plane from the physical apertures that act on signals in various LCT or equivalently FRT domains. However, since we have seen that these windows block or pass the space-frequency support of the signal in a manner very similar to how apertures block or pass the physical signals, we can also speak of space-frequency apertures or phase-space apertures.

\section{REFERENCES}

1. H. M. Ozaktas, Z. Zalevsky, and M. A. Kutay, The Fractional Fourier Transform with Applications in Optics and Signal Processing (Wiley, 2001).

2. A. W. Lohmann, R. G. Dorsch, D. Mendlovic, Z. Zalevsky, and C. Ferreira, "Space-bandwidth product of optical signals and systems," J. Opt. Soc. Am. A 13, 470-473 (1996).

3. G. Toraldo di Francia, "Resolving power and information," J. Opt. Soc. Am. 45, 497-499 (1955).

4. D. Gabor, "Light and information," in Progress In Optics, E. Wolf, ed. (Elsevier, 1961), Vol. I, Chap. 4, pp. 109-153.

5. A. W. Lohmann, "The space-bandwidth product, applied to spatial filtering and holography," Research paper RJ-438 (IBM San Jose Research Laboratory, 1967).

6. G. Toraldo di Francia, "Degrees of freedom of an image," J. Opt. Soc. Am. 59, 799-803 (1969).

7. F. Gori and G. Guattari, "Effects of coherence on the degrees of freedom of an image," J. Opt. Soc. Am. 61, 36-39 (1971).

8. F. Gori and G. Guattari, "Shannon number and degrees of freedom of an image," Opt. Commun. 7, 163-165 (1973).

9. F. Gori and G. Guattari, "Degrees of freedom of images from point-like-element pupils," J. Opt. Soc. Am. 64, 453-458 (1974).

10. F. Gori, S. Paolucci, and L. Ronchi, "Degrees of freedom of an optical image in coherent illumination, in the presence of aberrations," J. Opt. Soc. Am. 65, 495-501 (1975).

11. L. Ronchi and F. Gori, "Degrees of freedom for spherical scatterers," Opt. Lett. 6, 478-480 (1981).

12. A. Starikov, "Effective number of degrees of freedom of partially coherent sources," J. Opt. Soc. Am. 72, 1538-1544 (1982).

13. G. Newsam and R. Barakat, "Essential dimension as a welldefined number of degrees of freedom of finite-convolution operators appearing in optics," J. Opt. Soc. Am. A 2, 2040-2045 (1985).

14. A. W. Lohmann, Optical Information Processing, Lecture notes (Optik+Info, 1986).

15. R. Piestun and D. A. B. Miller, "Electromagnetic degrees of freedom of an optical system," J. Opt. Soc. Am. A 17, 892-902 (2000).
16. A. Stern and B. Javidi, "Shannon number and information capacity of three-dimensional integral imaging," J. Opt. Soc. Am. A 21, 1602-1612 (2004).

17. R. Solimene and R. Pierri, "Number of degrees of freedom of the radiated field over multiple bounded domains," Opt. Lett. 32, 3113-3115 (2007).

18. F. S. Oktem and H. M. Ozaktas, "Exact relation between continuous and discrete linear canonical transforms," IEEE Signal Process. Lett 16, 727-730 (2009).

19. F. S. Oktem and H. M. Ozaktas, "Equivalence of linear canonical transform domains to fractional Fourier domains and the bicanonical width product: a generalization of the space-bandwidth product," J. Opt. Soc. Am. A 27, 1885-1895 (2010).

20. F. S. Oktem, "Signal representation and recovery under partial information, redundancy, and generalized finite extent constraints," Master's thesis (Bilkent University, 2009).

21. D. Mendlovic and A. Lohmann, "Space-bandwidth product adaptation and its application to superresolution: fundamentals," J. Opt. Soc. Am. A 14, 558-562 (1997).

22. Z. Zalevsky, D. Mendlovic, and A. Lohmann, "Understanding superresolution in wigner space," J. Opt. Soc. Am. A 17, 2422-2430 (2000).

23. P. Catrysse and B. Wandell, "Optical efficiency of image sensor pixels,” J. Opt. Soc. Am. A 19, 1610-1620 (2002).

24. M. J. Bastiaans and T. Alieva, "Classification of lossless firstorder optical systems and the linear canonical transformation," J. Opt. Soc. Am. A 24, 1053-1062 (2007).

25. T. Alieva and M. J. Bastiaans, "Properties of the linear canonical integral transformation,” J. Opt. Soc. Am. A 24, 3658-3665 (2007).

26. A. Stern, "Uncertainty principles in linear canonical transform domains and some of their implications in optics," J. Opt. Soc. Am. A 25, 647-652 (2008)

27. B. Barshan, M. A. Kutay, and H. M. Ozaktas, "Optimal filtering with linear canonical transformations," Opt. Commun. 135, 32-36 (1997)

28. J. Rodrigo, T. Alieva, and M. Calvo, "Gyrator transform: properties and applications," Opt. Express 15, 2190-2203 (2007).

29. J. J. Healy and J. T. Sheridan, "Cases where the linear canonical transform of a signal has compact support or is band-limited," Opt. Lett. 33, 228-230 (2008).

30. H. M. Ozaktas, M. A. Kutay, and C. Candan, "Fractional Fourier transform," in Transforms and Applications Handbook, A. D. Poularikas, ed. (CRC, 2010), pp. 14-1-14-28.

31. K. B. Wolf, "Construction and properties of canonical transforms," in Integral Transforms in Science and Engineering (Plenum, 1979), Chap. 9.

32. H. M. Ozaktas, A. Koç, I. Sari, and M. A. Kutay, "Efficient computation of quadratic-phase integrals in optics," Opt. Lett. 31, 35-37 (2006).

33. A. Koc, H. M. Ozaktas, C. Candan, and M. A. Kutay, "Digital computation of linear canonical transforms," IEEE Trans. Signal Process. 56, 2383-2394 (2008).

34. T. Alieva and M. J. Bastiaans, "Alternative representation of the linear canonical integral transform," Opt. Lett. 30, 3302-3304 (2005).

35. M. J. Bastiaans and T. Alieva, "Synthesis of an arbitrary ABCD system with fixed lens positions," Opt. Lett. 31, 2414-2416 (2006).

36. J. A. Rodrigo, T. Alieva, and M. L. Calvo, "Optical system design for orthosymplectic transformations in phase space," J. Opt. Soc. Am. A 23, 2494-2500 (2006).

37. H. M. Ozaktas and M. F. Erden, "Relationships among ray optical, Gaussian beam, and fractional Fourier transform descriptions of first-order optical systems," Opt. Commun. 143, 75-86 (1997).

38. H. Ozaktas, S. Ark, and T. Coşkun, "Fundamental structure of fresnel diffraction: natural sampling grid and the fractional Fourier transform," Opt. Lett. 36, 2524-2526 (2011).

39. H. Ozaktas, S. Ark, and T. Coşkun, "Fundamental structure of Fresnel diffraction: longitudinal uniformity with respect to fractional Fourier order," Opt. Lett. 37, 103-105 (2012).

40. L. Cohen, Integral Time-Frequency Analysis (Prentice-Hall, 1995). 
41. M. J. Bastiaans, "Applications of the Wigner distribution function in optics," in The Wigner Distribution: Theory and Applications in Signal Processing, W. Meckenbrauker, ed. (Elsevier, 1997), pp. 375-426.

42. G. Forbes, V. Manko, H. Ozaktas, R. Simon, and K. Wolf, "Wigner distributions and phase space in optics," J. Opt. Soc. Am. A 17, $2274(2000)$.

43. J. Maycock, C. McElhinney, B. Hennelly, T. Naughton, J. McDonald, and B. Javidi, "Reconstruction of partially occluded objects encoded in three-dimensional scenes by using digital holograms," Appl. Opt. 45, 2975-2985 (2006).

44. H. M. Ozaktas and O. Aytur, "Fractional Fourier domains," Signal Process. 46, 119-124 (1995).

45. L. Cohen, "Time-frequency distributions-a review," Proc. IEEE 77, 941-981 (1989).

46. H. M. Ozaktas, B. Barshan, D. Mendlovic, and L. Onural, "Convolution, filtering, and multiplexing in fractional Fourier domains and their relation to chirp and wavelet transforms," J. Opt. Soc. Am. A 11, 547-559 (1994).

47. D. Mendlovic, A. Lohmann, and Z. Zalevsky, "Space-bandwidth product adaptation and its application to superresolution: examples,” J. Opt. Soc. Am. A 14, 563-567 (1997).

48. K. Wolf, D. Mendlovic, and Z. Zalevsky, "Generalized Wigner function for the analysis of superresolution systems," Appl. Opt. 37, 4374-4379 (1998).
49. Z. Zalevsky, N. Shamir, and D. Mendlovic, "Geometrical superresolution in infrared sensor: experimental verification," Opt. Eng. 43, 1401-1406 (2004).

50. Z. Zalevsky, V. Mico, and J. Garcia, "Nanophotonics for optical super resolution from an information theoretical perspective: a review," J. Nanophoton. 3, 032502 (2009).

51. J. Lindberg, "Mathematical concepts of optical superresolution," J. Opt. 14, 083001 (2012)

52. L. Xu, X. Peng, Z. Guo, J. Miao, and A. Asundi, "Imaging analysis of digital holography," Opt. Express 13, 2444-2452 (2005).

53. M. Testorf and A. Lohmann, "Holography in phase space," Appl. Opt. 47, A70-A77 (2008).

54. U. Gopinathan, G. Pedrini, B. Javidi, and W. Osten, "Lensless 3D digital holographic microscopic imaging at vacuum UV wavelength," J. Disp. Technol. 6, 479-483 (2010).

55. D. Claus, D. Iliescu, and P. Bryanston-Cross, "Quantitative space-bandwidth product analysis in digital holography," Appl. Opt. 50, H116-H127 (2011).

56. B. H. Walker, Optical Design for Visual Systems (SPIE, 2000).

57. B. Hennelly and J. Sheridan, "Optical encryption and the space bandwidth product," Opt. Commun. 247, 291-305 (2005).

58. J. Healy and J. Sheridan, "Bandwidth, compact support, apertures and the linear canonical transform in ABCD systems," Proc. SPIE 6994, 69940W (2008). 